EGU21-13463

EGU General Assembly 2021

(c) Author(s) 2021. This work is distributed under

the Creative Commons Attribution 4.0 License.

\title{
On the Use of Short-Time Fourier Transform for the Analysis of Tree Root Systems using Ground Penetrating Radar
}

\author{
Livia Lantini ${ }^{1}$, Fabio Tosti ${ }^{1}$, Luca Bianchini Ciampoli ${ }^{2}$, and Amir M. Alani ${ }^{1}$ \\ ${ }^{1}$ School of Computing and Engineering, University of West London, London, United Kingdom of Great Britain - England, \\ Scotland, Wales (Livia.Lantini@uwl.ac.uk, Fabio.Tosti@uwl.ac.uk, Amir.Alani@uwl.ac.uk) \\ 2Department of Engineering, Roma Tre University, Rome, Italy (luca.bianchiniciampoli@uniroma3.it)
}

Monitoring and protecting natural assets is increasingly important today, as aggressive pathogens are negatively impacting the trees' survival. In this regard, root systems are affected by fungal infections that cause roots' rot and eventually lead to trees' death. Such disease can spread rapidly to the adjacent trees and affect larger areas. Since these decays generally do not display visible signs, early identification is the key to tree preservation.

Within this context, non-destructive testing (NDT) methods are becoming popular, being more versatile than destructive methods. Specifically, ground penetrating radar (GPR) is emerging as an accurate geophysical method for tree root mapping. Recent research has focused on implementing automated algorithms for 3D root mapping, improving root detection through advanced GPR signal processing and the estimation of tree roots' mass density [1]. Also, recent studies have proven that GPR is effective in mapping the root system's architecture of street trees [2].

The present research reports the preliminary results of an experimental study, conducted to investigate the feasibility of a novel tree root assessment methodology based on the analysis of GPR data both in time and frequency domain. To this end, data were processed using a short-time Fourier transform (STFT) approach [3], which allows the evaluation of how the frequency spectrum changes across the signal propagation time window. The suggested processing system may be implemented for expeditious analyses or on trees challenging to access, such as in urban environments, where more comprehensive survey methods are not applicable. The objectives of this study, therefore, are to investigate how different features (i.e., roots, layers) affect the timefrequency analysis of GPR data, and to identify recurring patterns in the results to set a coherent data processing methodology.

Results' interpretation has shown the viability of the presented approach in recognising the influence of different features on the analysis of GPR data as it changes over time. This also allowed the detection of recurring patterns in the analysed data, proving that this method is worthy of further investigations.

Acknowledgements

The authors would like to express their sincere thanks and gratitude to the following trusts, 
charities, organisations and individuals for their generosity in supporting this project: Lord Faringdon Charitable Trust, The Schroder Foundation, Cazenove Charitable Trust, Ernest Cook Trust, Sir Henry Keswick, Ian Bond, P. F. Charitable Trust, Prospect Investment Management Limited, The Adrian Swire Charitable Trust, The John Swire 1989 Charitable Trust, The Sackler Trust, The Tanlaw Foundation, and The Wyfold Charitable Trust.

\section{References}

[1] Lantini, L., Tosti, F., Giannakis, I., Zou, L., Benedetto, A. and Alani, A. M., 2020. "An Enhanced Data Processing Framework for Mapping Tree Root Systems Using Ground Penetrating Radar," Remote Sensing 12(20), 3417.

[2] Lantini, L., Alani, A., Giannakis, I., Benedetto, A. and Tosti, F., 2020. "Application of ground penetrating radar for mapping tree root system architecture and mass density of street trees," Advances in Transportation Studies (3), 51-62.

[3] Bianchini Ciampoli, L., Calvi, A. and D'Amico, F., 2019. "Railway Ballast Monitoring by GPR: A Test Site Investigation," Remote Sensing 11(20), 238 